\title{
Two Ising-like magnetic excitations in a single-layer cuprate superconductor
}

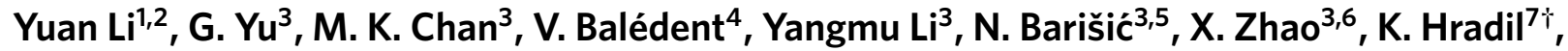 \\ R. A. Mole ${ }^{8 \dagger}$, Y. Sidis ${ }^{4}$, P. Steffens ${ }^{9}$, P. Bourges ${ }^{4}$ and M. Greven ${ }^{3 \star}$
}

\begin{abstract}
There exists increasing evidence that the phase diagram of the high-transition temperature $\left(T_{c}\right)$ cuprate superconductors is controlled by a quantum critical point. According to one distinct theoretical proposal, on decreasing the hole-carrier concentration a transition occurs to an ordered state with two circulating orbital currents per $\mathrm{CuO}_{2}$ square. $\mathrm{Bel} \mathrm{cw}$ the 'pseudogap' temperature $T^{*}\left(T^{*}>T_{c}\right)$, the theory predicts a discrete order parameter and two weakly-dispersive magnetic excitations in structurally simple compounds which should be measurable by neutron scattering. Indeed, novel magnetic order and one such excitation were recently observed. Here, we demonstrate for tetragonal $\mathrm{HgBa}_{2} \mathrm{CuO}_{4+\delta}$ the existence of a second excitation with local character, consistent with the theory. The excitations mix with conventional antiferromagnetic fluctuations, which points towards a unifying picture of magnetism in the cuprates that will probably require a multiband description.
\end{abstract}

t $t$ is widely agreed that attaining a thorough understanding of the peculiar electronic and magnetic properties in the pseudogap regime of the cuprates would constitute a major leap towards solving the high- $T_{\mathrm{c}}$ problem. A pivotal and intensely debated question has been whether this regime is a genuine new phase of matter and, if so, what symmetries are broken at the pseudogap temperature $T^{*}$ (refs $1-4$ ). There is mounting evidence that $T^{*}$ indeed marks a transition into a novel electronic phase in which time-reversal symmetry is broken ${ }^{5-10}$ and, in compounds with relatively high maximal transition temperatures $\left(T_{\mathrm{c}, \max }>90 \mathrm{~K}\right.$ at the optimal hole concentration $p_{\mathrm{opt}} \approx 16 \%$ per planar $\mathrm{Cu}$ atom), translational symmetry is preserved ${ }^{6-8,11}$.

Neutron scattering is a powerful probe of magnetic correlations and has shed much light on the high- $T_{\mathrm{c}}$ problem. In the superconducting doping regime, magnetic neutron scattering experiments have been carried out mostly near the two-dimensional (2D) wave vector $\mathbf{q}_{\mathrm{AF}}$ that characterizes the antiferromagnetic order of the undoped Mott-insulating parent compounds ${ }^{12-20}$. A spin-1 'resonance' excitation ${ }^{13,15-17,21}$ is observed at $\mathbf{q}_{\mathrm{AF}}$ in the superconducting state, between nearly temperature-independent spin fluctuations at higher energy and a magnetic gap at lower energy. This phenomenon has been regarded as indicative of a magneticfluctuation-driven superconducting mechanism ${ }^{22,23}$. On the other hand, recent measurements of the $T_{c, \max }>90 \mathrm{~K}$ compounds $\mathrm{YBa}_{2} \mathrm{Cu}_{3} \mathrm{O}_{6+\delta}$ (YBCO; ref. 6) and $\mathrm{HgBa}_{2} \mathrm{CuO}_{4+\delta}$ ( $\mathrm{Hg} 1201$; refs 7,8) revealed a novel kind of magnetic order (broken time-reversal symmetry) below $T^{*}$ that is characterized by the wave vector $q=0$ (preserved lattice translational symmetry). The measurements were motivated by the distinct theoretical proposal that magnetism due to orbital charge currents (rather than local spin moments) lies at the heart of the cuprate phase diagram ${ }^{1}$. The subsequent discovery of a prominent magnetic excitation in Hg1201, that also appears below $T^{*}$ and is centred at $q=0$, seems to be the first dynamic fingerprint of this pseudogap magnetism ${ }^{24}$. However, it has remained largely elusive if and how the antiferromagnetism and the pseudogap magnetism are related. Here we use inelastic neutron scattering to further determine the excitation spectrum associated with the latter. Our new results for $\mathrm{Hg} 1201$ reveal a second weakly dispersive magnetic excitation branch, as predicted theoretically ${ }^{25,26}$, as well as an intriguing mixing with the antiferromagnetic fluctuations near $\mathbf{q}_{\mathrm{AF}}$ that is not yet explained theoretically.

Hg1201 has a simple tetragonal crystal structure, exhibits the highest value of $T_{c \text { max }}(\approx 96 \mathrm{~K})$ of all single-layer cuprates (one $\mathrm{CuO}_{2}$ layer per primitive cell), and is thought to be relatively free of disorder effects ${ }^{27,28}$. Sizeable crystals of Hg1201 have become available only in recent years ${ }^{29}$ and enabled initial neutron scattering experiments $\mathrm{s}^{7,8,24,30,31}$. Our underdoped $\left(T_{\mathrm{c}}=65 \mathrm{~K}\right.$, $T^{*} \approx 330 \mathrm{~K}$, mass $=1.8 \mathrm{~g}$; denoted UD65) and a nearly optimally doped $\left(T_{\mathrm{c}}=95 \mathrm{~K}, T^{*} \approx 210 \mathrm{~K}\right.$, mass $=2.0 \mathrm{~g}$; denoted OP95) samples ${ }^{24}$ were measured with both spin-polarized and unpolarized neutrons. Scattering wave vectors are quoted as $\mathbf{Q}=H \mathbf{a}^{*}+K \mathbf{b}^{*}+$ $L \mathbf{c}^{*} \equiv(H, K, L)$ in units of the reciprocal lattice vectors (r.l.u.), with typical room-temperature values $a^{*}=b^{*}=1.614 \AA^{-1}$ and $c^{*}=0.657 \AA^{-1}$. Further experimental details are provided in the Supplementary Information.

We first provide evidence for magnetic excitations below $T^{*}$ from measurements with unpolarized neutrons. Figure $1 \mathrm{a}-\mathrm{c}$ shows energy scans at various locations in the first 2D Brillouin zone. As both nuclear and magnetic scattering contribute to the intensity, we use the intensity difference between the lowest temperature and

\footnotetext{
${ }^{1}$ Department of Physics, Stanford University, Stanford, California 94305, USA, ${ }^{2}$ Max Planck Institute for Solid State Research, 70569 Stuttgart, Germany, ${ }^{3}$ School of Physics and Astronomy, University of Minnesota, Minneapolis, Minnesota 55455, USA, ${ }^{4}$ Laboratoire Léon Brillouin, CEA-CNRS, CEA-Saclay, 91191 Gif sur Yvette, France, ${ }^{5} 1$. Physikalisches Institut, Universität Stuttgart, 70550 Stuttgart, Germany, ${ }^{6}$ State Key Lab of Inorganic Synthesis and Preparative Chemistry, College of Chemistry, Jilin University, Changchun 130012, China, ${ }^{7}$ Institut für Physikalische Chemie, Universität Göttingen, 37077 Göttingen, Germany, ${ }^{8}$ Forschungsneutronenquelle Heinz Maier-Leibnitz, 85747 Garching, Germany, ${ }^{9}$ Institut Laue Langevin, 38042 Grenoble CEDEX 9 , France. ${ }^{\dagger}$ Present addresses: X-ray Center, Vienna University of Technology, 1060 Vienna, Austria (K.H.); The Bragg Institute, Australian Nuclear Science and Technology Organization, Locked Bag 2001, Kirrawee DC, NSW 2232, Australia (R.A.M.). ${ }^{\star}$ e-mail: greven@physics.umn.edu.
} 


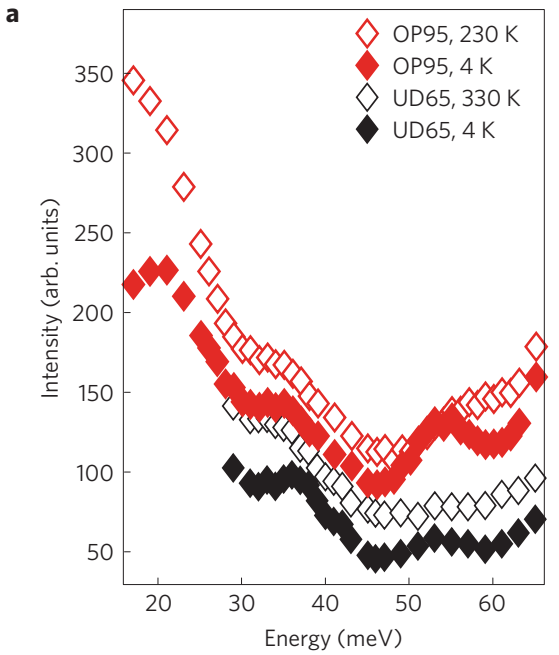

d

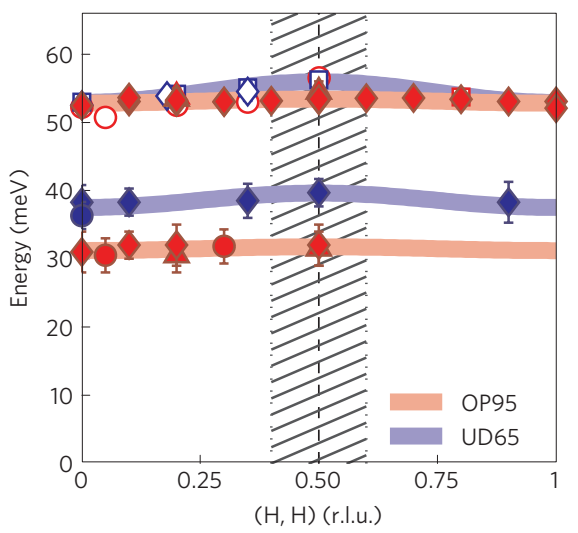

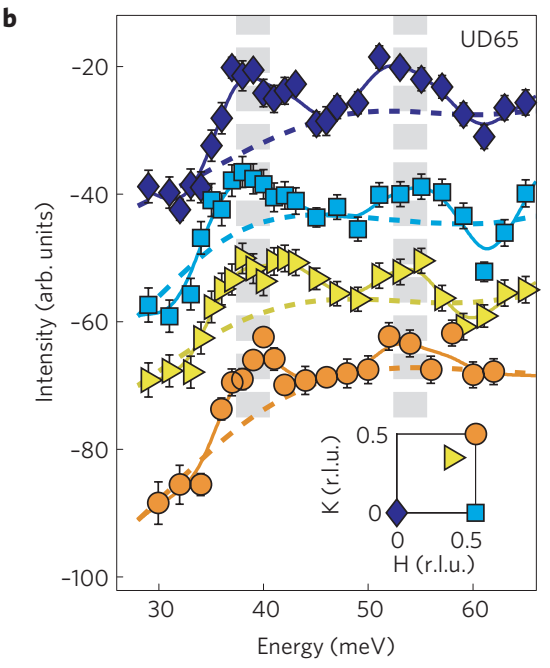
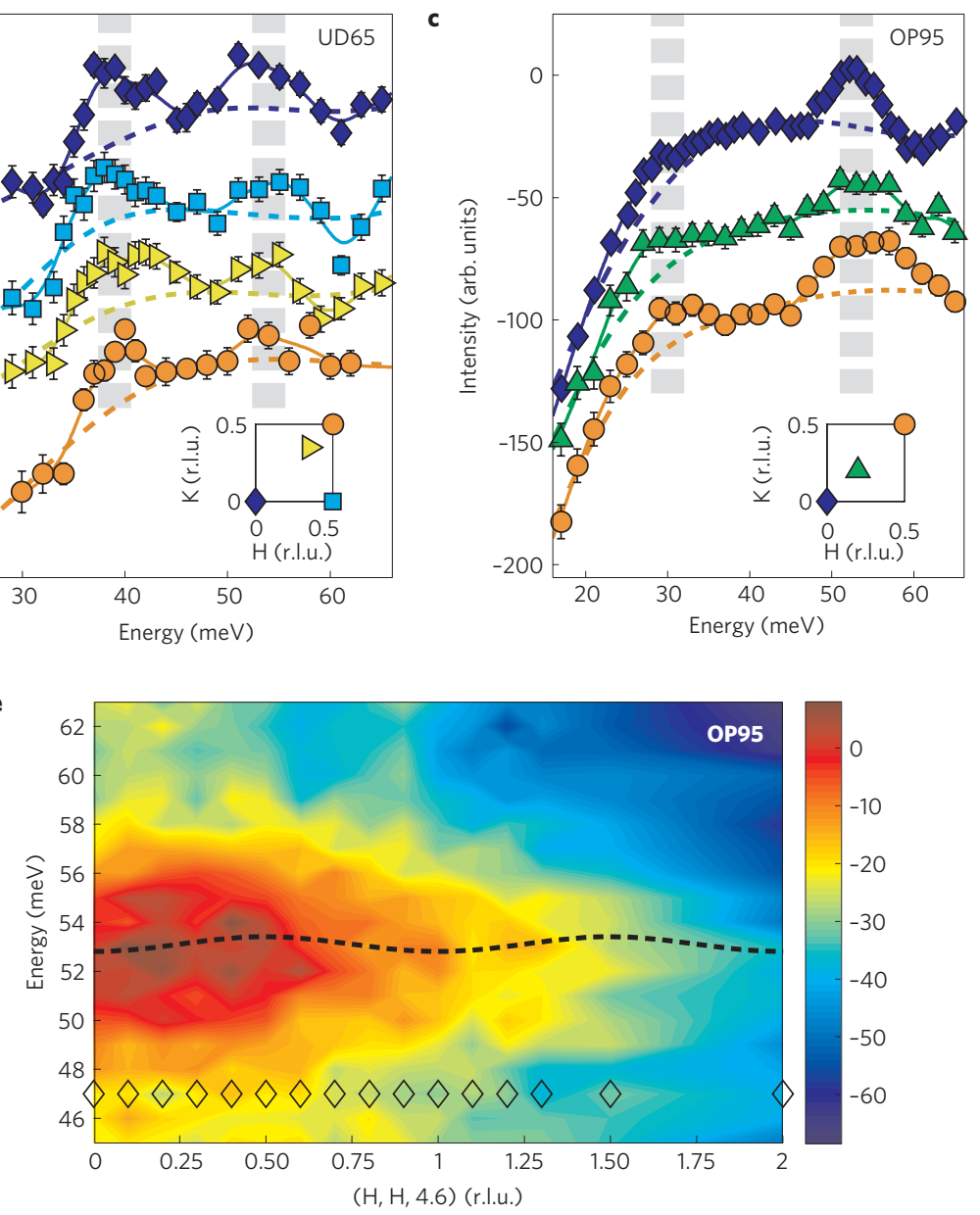

Figure 1 | Observation of two excitation branches. a, Unpolarized inelastic neutron scattering data at $\mathbf{Q}=(0,0,4.6)$. The high-energy $(\sim 53 \mathrm{meV})$ magnetic excitation reported in ref. 24 is evident from the $4 \mathrm{~K}$ data; the low-energy excitation is difficult to discern from the raw spectra because of phonons nearby. $\mathbf{b}$, Intensity difference between $4 \mathrm{~K}$ and $330 \mathrm{~K}$ (top three) and between $4 \mathrm{~K}$ and $300 \mathrm{~K}$ (bottom) for UD65 $\left(T^{*} \approx 330 \mathrm{~K}\right.$; ref. 24$)$ at $\mathbf{Q}=(0,0,4.6)$, $(0.5,0,4.6),(0.35,0.35,4.6)$ and $(0.5,0.5,4.4)$, from top to bottom. The bottom data set was obtained with better energy resolution ( $\sim 4$ meV (FWHM) at $\omega=40 \mathrm{meV}$, compared with $\sim 6 \mathrm{meV}$ for the rest). c, Intensity difference between $4 \mathrm{~K}$ and $230 \mathrm{~K}$ (top) and between $4 \mathrm{~K}$ and $200 \mathrm{~K}$ (bottom two, measured on a different spectrometer and rescaled for comparison) for OP95 ( $T^{*} \approx 210 \mathrm{~K}$; ref. 24) at $\mathbf{Q}=(0,0,4.6),(0.2,0.2,4.4)$ and $(0.5,0.5,4.4)$, from top to bottom. In $\mathbf{b}$ and $\mathbf{c}$, the solid lines are guides to the eye, and the data are offset for clarity (top data sets are without offset). The magnetic signal is superposed on a baseline (dashed lines) that is more negative at lower energies owing to the stronger increase of phonon scattering towards high temperatures. The insets indicate the measured 2D momentum positions. d, Dispersion along $[H, H]$ of the two excitations observed at the two doping levels. Open symbols are data reported in ref. 24. Different symbols indicate on which spectrometers the measurements were performed (diamond: IN8; circle: IN2O; square: PUMA; triangle: 2 T). The hatched area indicates where antiferromagnetic spin fluctuations are expected. Error bars in a-c represent statistical uncertainty ( $1 \mathrm{s.d}$.), and in $\mathbf{d}$ the confidence range for the estimated energies. $\mathbf{e}$, Colour representation of the intensity difference between $4 \mathrm{~K}$ and $230 \mathrm{~K}$ for the high-energy excitation (dashed line) of OP95. Diamond symbols indicate the momentum positions where energy scans were performed.

a high temperature (close to $T^{*}$ ) to extract magnetic signals, on the basis of the expectation that phonon intensity decreases on cooling, whereas magnetic intensity increases. In particular, for UD65, this method clearly reveals the presence of two weakly dispersive excitation branches throughout the entire Brillouin zone, with approximate energies of 38 and $54 \mathrm{meV}$ (Fig. 1b). The branch near $54 \mathrm{meV}$ was the subject of our previous study, and its magnetic origin was verified with spin-polarized neutrons ${ }^{24}$. The result in Fig. 1e further confirms this conclusion: apart from an enhancement near $\mathbf{q}_{\mathrm{AF}}$ due to the presence of conventional antiferromagnetic fluctuations (Supplementary Figs S1a and S2), the signal gradually decreases towards large in-plane momentum transfer, consistent with a magnetic origin. A phonon-based interpretation is further ruled out by the comparison between the scattering at $(0,0,4.6)$ and $(2,2,4.6)$, because the phonon dynamic structure factor at $(0,0,4.6)$ cannot be larger than at $(2,2,4.6)$, yet the intensity at the former position is clearly larger (Supplementary Fig. S3). A similar decrease of intensity with increasing $Q$ is found for the low-energy excitation branch in UD65 (see Fig. 5a later and Supplementary Fig. S6a), implying that it is also of magnetic origin. Figure $1 \mathrm{~d}$ summarizes our results for the dispersion of the two branches along $[H, H]$.

Although the presence of a low-energy excitation is not as evident for OP95 as for UD65, there is a clear difference between the data in Figs $1 \mathrm{~b}$ and 1c: unlike for UD65, for OP95 there is no peak at $\sim 38 \mathrm{meV}$, but instead a 'shoulder' near $31 \mathrm{meV}$. This is best seen in Fig. 2a by comparing the ' $4 \mathrm{~K}-330 \mathrm{~K}$ ' intensity difference for both samples, measured at $(0,0,4.6)$ under nearly identical experimental conditions. Given the rather small difference in oxygen concentration between OP95 and UD65 $(\Delta \delta \sim 0.03$, assuming each oxygen dopes two holes), the difference in the data is rather unlikely to be due to phonons and more naturally explained by a shift of the excitation from $\sim 38 \mathrm{meV}$ in UD65 to $\sim 31 \mathrm{meV}$ in OP95, reflecting a doping dependence of the underlying magnetism. 


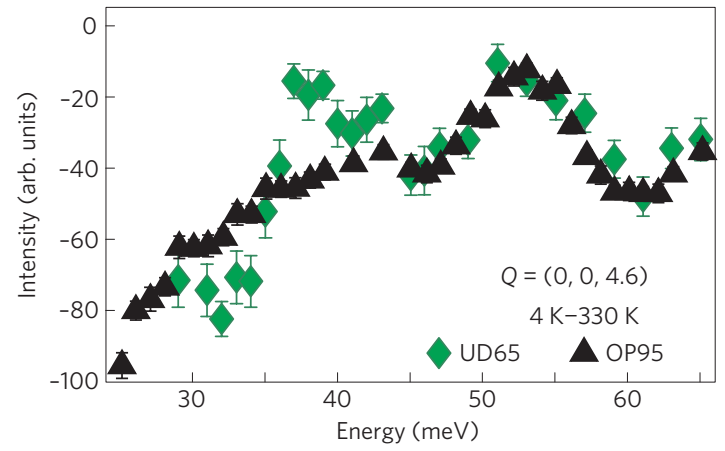

b

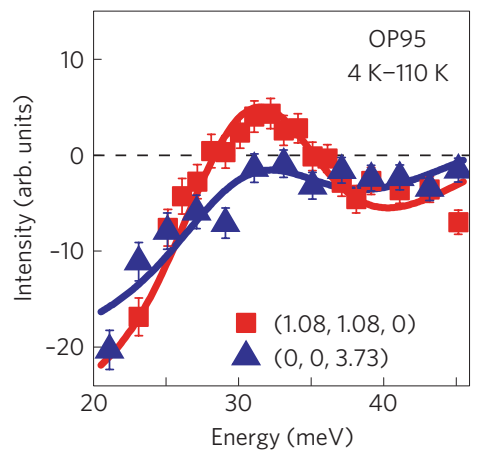

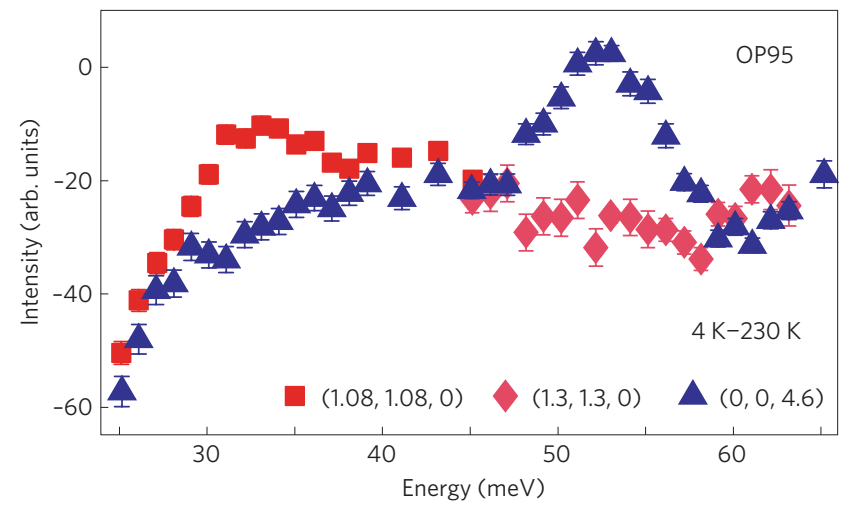

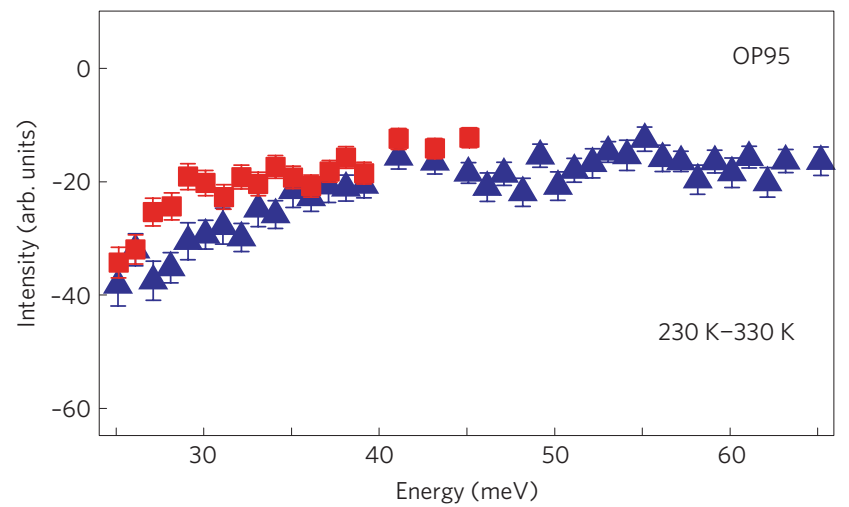

Figure 2 | Doping and momentum dependence of intensity. a, Doping dependence of low-energy excitation revealed by net intensity (' 4 K-330 K') for both samples. The measurements were performed on the same spectrometer with a similar configuration. The UD65 data are rescaled to the high-energy ( $~ 53$ meV) signal of the OP95 data. b, Intensity difference between $4 \mathrm{~K}$ and $110 \mathrm{~K}$ measured with $\mathbf{Q} \| c$ and $\mathbf{Q} \| a b$ for the low-energy excitation in sample OP95. The magnitudes of the two momenta $(1.08,1.08,0)$ and $(0,0,3.73)$ are identical. By using a low reference temperature of $110 \mathrm{~K}$, the increase of the magnetic signal at $\sim 31 \mathrm{meV}$ towards $4 \mathrm{~K}$ overcomes the decrease in phonon scattering, leading to a net intensity increase near 31 me $\mathrm{V}$ for $\mathbf{Q}=(1.08,1.08,0)$. c, Intensity difference between $4 \mathrm{~K}$ and $230 \mathrm{~K}$ from energy scans for OP95. In contrast to $\mathbf{b}$, the low-energy excitation at $\mathbf{Q}=(0,0,4.6)$ is difficult to discern from these data because of the baseline slope due to phonons. The magnitudes of the momenta $(1.3,1.3,0)$ and $(0,0,4.6)$ are identical. $\mathbf{d}$, Intensity difference between $230 \mathrm{~K}$ and $330 \mathrm{~K}$ for OP95. The $\mathbf{Q}=(1.08,1.08,0)$ data lie above $\mathbf{Q}=(0,0$, 4.6) because the baseline due to phonons is less negative at smaller $Q$, and therefore the difference is not necessarily a low-energy magnetic signal. Error bars represent statistical uncertainty (1 s.d.).

The presence of a magnetic signal at $\sim 31 \mathrm{meV}$ in OP95 is further supported by the data in Fig. 2c, which reveal that the intensities of the two excitations depend on the momentum transfer direction in a peculiar, opposite fashion. It was previously found that the highenergy excitation becomes indiscernible when $\mathbf{Q}$ is parallel to the $\mathrm{CuO}_{2}$ planes (Supplementary Fig. S4 of ref. 24), which is confirmed in Fig. 2c. Conversely, although non-zero intensity is observed for the low-energy excitation for $\mathbf{Q} \| c$, higher intensity is observed for $\mathbf{Q} \| a b$ with both unpolarized (Fig. 2c) and polarized neutrons (Fig. 4b,c). The low-energy features at both $\mathbf{Q}$ positions are more clearly observed from the ' $4 \mathrm{~K}-110 \mathrm{~K}$ ' intensity difference (Fig. 2b), because the lower reference temperature keeps the variation in phonon scattering to a minimum. The opposite momentum dependence of the intensities implies that the two excitation branches are associated with fluctuations in perpendicular directions, either purely in the magnetic degrees of freedom, or in conjunction with lattice vibrations. However, without an extensive study of the neutron spin-polarization dependence of the signal beyond the present work (especially of the low-energy branch with $\mathbf{Q} \| a b$, which would allow for a differentiation between magnetic fluctuations parallel and perpendicular to the copper-oxygen planes) a conclusive explanation of this phenomenon is unreachable. Here we simply regard it as empirical evidence that the two branches have the same physical origin. This is further evinced by the fact that the excitations exhibit similar intensity amplitudes (Figs 1b,c and 2a) and temperature dependences (Fig. 3), with an onset temperature consistent with $T^{*}$ determined from resistivity and neutron diffraction ${ }^{21}$. No well-defined magnetic signal is observed in the raw data above $T^{*}$ (Fig. 1a) or in the intensity difference for temperatures above $T^{*}$ (Fig. 2d). Together with the fact that the excitations emanate from $q=0$ (Fig. 1e), this demonstrates that they are associated with the $q=0$ magnetic order.

We used spin-polarized neutrons (see Supplementary Information for a detailed description of the method) to further verify the magnetic origin of the low-energy excitation branch. Such measurements are extremely difficult, not only because of the much reduced neutron flux, but also because a large part of the background intensity arises from incoherent scattering and cannot be suppressed further in spin-flip measurements. Moreover, imperfect shielding leads to an additional (small) background intensity which is not negligible compared to the weak signal strength in the polarized measurements. Altogether, this results in a much reduced signal, but not necessarily an improved signal-to-background ratio compared to unpolarized measurements, hence extremely long counting times are required (see Supplementary Fig. S4 for a comparison between polarized and unpolarized measurements).

In Fig. $4 a-c$, the intensity difference between low and high temperatures for OP95, measured in the spin-flip scattering geometry, shows a peak at $\sim 31 \mathrm{meV}$ near the $2 \mathrm{D}$ zone centre (Fig. $4 \mathrm{~b}$ ) and also for $L=0$ (Fig. 4c), consistent with the unpolarized results (Figs 1c, $2 \mathrm{a}, \mathrm{c}, 3 \mathrm{~b}, \mathrm{c})$. As no prominent nuclear scattering feature is observed in the non-spin-flip geometry (Fig. 4d,e), the experiment's flipping 


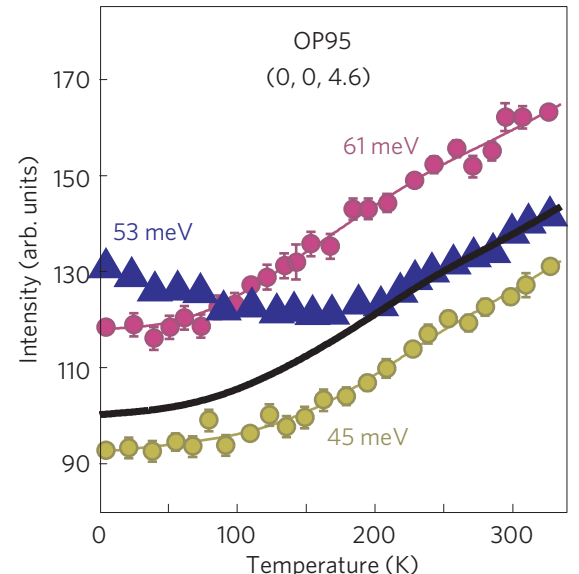

b

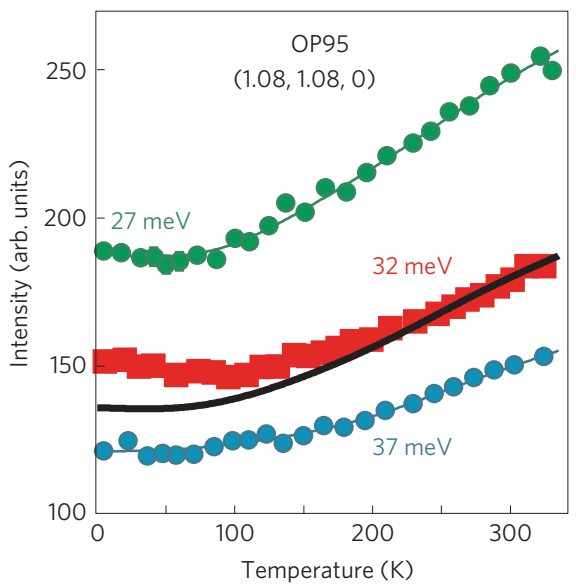

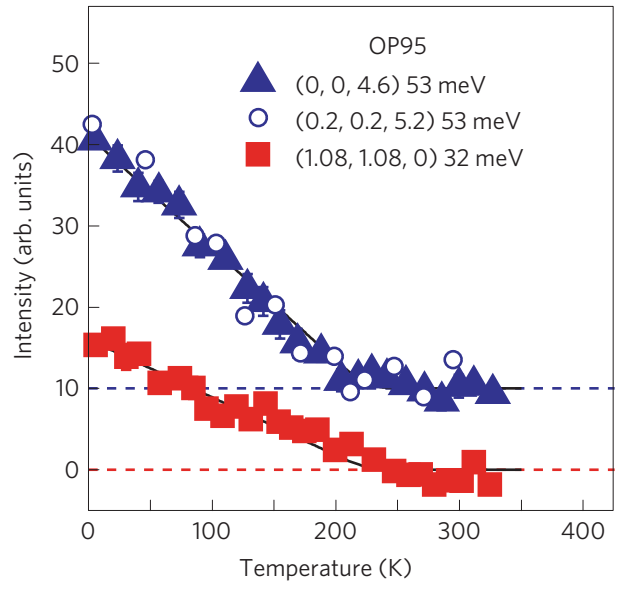

d

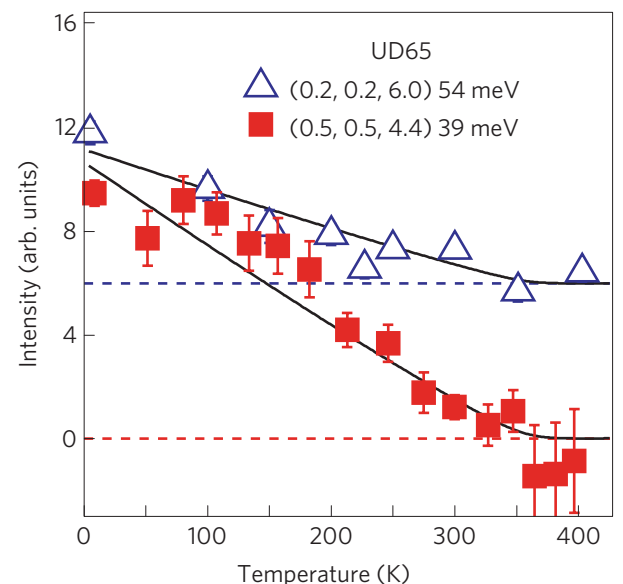

Figure 3 | Temperature dependence. a,b, Temperature dependence of intensities at the indicated energies and momenta for OP95. c, The temperature

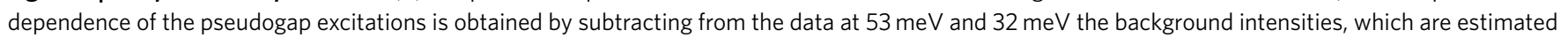
as the average of the smoothed temperature dependences (thin lines in $\mathbf{a}, \mathbf{b}$ ) at higher and lower energy with a vertical offset (black lines in $\mathbf{a}, \mathbf{b}$ ). $\mathbf{d}$, The same method is used to obtain the temperature dependence of the pseudogap excitations for UD65. Data in c and $\mathbf{d}$ are offset for clarity (dashed lines), and the open symbols are data reported in ref. 24. Solid lines are guides to the eye. Error bars represent statistical uncertainty (1 s.d.).

ratio of approximately 10 (which is high for inelastic scattering at these energies) ensures that the observed spin-flip signal is not due to polarization leakage. We note, on the other hand, that the data do not allow us to rule out a non-spin-flip contribution that is comparable in strength to the spin-flip signal. Hence, it is not impossible that the excitations contain an admixture with lattice vibrations.

A more stringent test of magnetic scattering uses the polarization dependence of any genuine magnetic signal: spin-flip scattering probes magnetic fluctuations perpendicular to both the momentum transfer, $\mathbf{Q}$, and the spin polarization of the incident neutrons, $\mathbf{S}$. As a result, the magnetic signal is maximized when $S$ is parallel to $\mathbf{Q}(\mathbf{S} \| \mathbf{Q})$, whereas all other scattering processes are independent of the orientation of $\mathbf{S}$. The purely magnetic signal can be extracted by taking the intensity $(I)$ combination: $2 \times I_{S \| Q}-I_{S \perp Q}-I_{S \| Z}$ (see Supplementary Information for details), where $\mathbf{S} \perp \mathbf{Q}$ and $\mathbf{S} \| \mathbf{Z}$ denote the two geometries in which $\mathbf{S}$ is perpendicular to $\mathbf{Q}$, horizontal and vertical, respectively. Based on the results in Fig. 4a,f, Fig. 4g therefore demonstrates the presence of magnetic intensity centred at $\sim 30 \mathrm{meV}$ (OP95) and $\sim 37 \mathrm{meV}$ (UD65), in excellent agreement with the unpolarized-neutron data in Figs $4 \mathrm{~h}, 1 \mathrm{~b}, \mathrm{c}$, and $2 \mathrm{a}-\mathrm{c}$. Although the individual errors in Fig. $4 \mathrm{~g}$ are relatively large, statistical analysis shows it to be a very robust result that the excitation (established to be present with unpolarized neutrons) is indeed predominantly magnetic (see Supplementary Information).

Our results provide valuable insight into the fundamental properties of the pseudogap magnetism. The very weak dispersion of about 5\% (Fig. 1d) and the absence of a Goldstone mode dispersing to zero energy at the ordering wave vector $q=0$ imply that the order parameter has discrete symmetry. The dispersion is even weaker than that of the classic local-moment Ising-like antiferromagnet $\mathrm{Rb}_{2} \mathrm{CoF}_{4}$, in which the spin excitations disperse by about $20 \%$ (ref. 32). Contrary to this model magnet, we observe two excitation branches rather than one. Together, these results suggest the presence of multiple scattering centres per $\mathrm{CuO}_{2}$ square (or $\mathrm{CuO}_{6}$ octahedron) and the need for a multi-band rather than a single-band theoretical description. The orbital-current theory, which is based on a multi-band Hamiltonian and makes the non-trivial prediction of two magnetic collective excitations in a single-layer system measurable via neutron scattering, seems to be able to explain our findings ${ }^{25,26,33}$. In this model, the weak dispersion is a direct consequence of the underlying discrete order parameter, whereas the non-degeneracy of the excitations has been suggested to be due to the nature of the ground and excitation states, which are quantum superpositions of four 'classical' degenerate orbital-current patterns ${ }^{26,33}$. This superposition has also been proposed to account for the peculiar experimental result that the magnetic moment direction is neither perpendicular nor parallel to the $\mathrm{CuO}_{2}$ layers ${ }^{6-8,34}$. On general grounds, mode softening is expected at high temperature and on approaching the quantum critical point. The former is not observed in our experiment and would require high-statistics energy scans at temperatures just below $T^{*}$. However, with increasing doping, we observe a clear softening of the low-energy branch. 
a

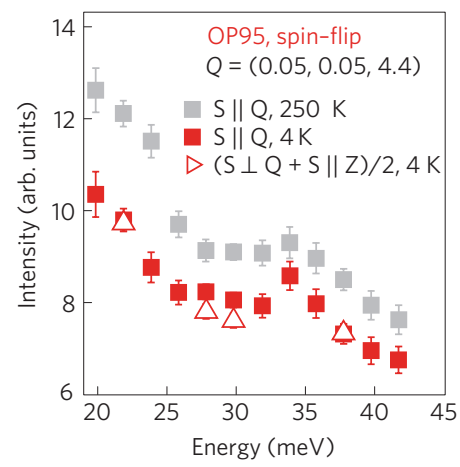

b
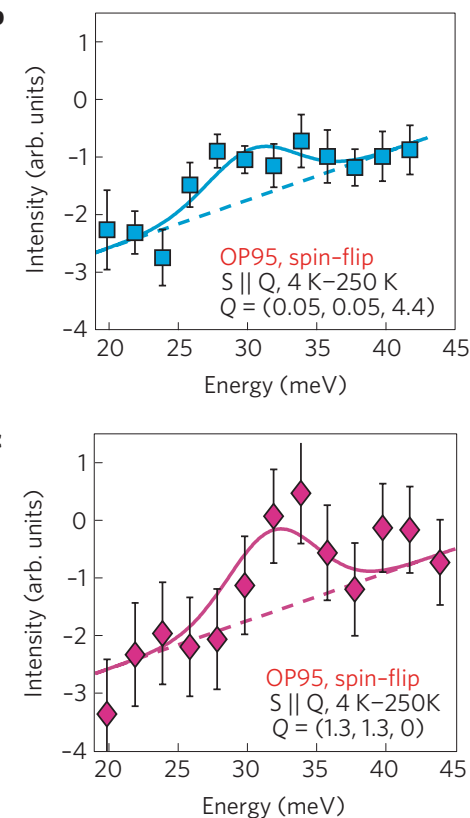

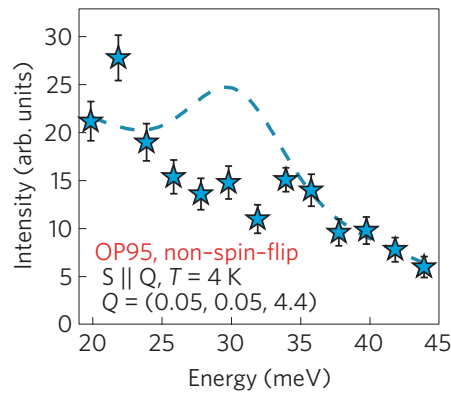

e

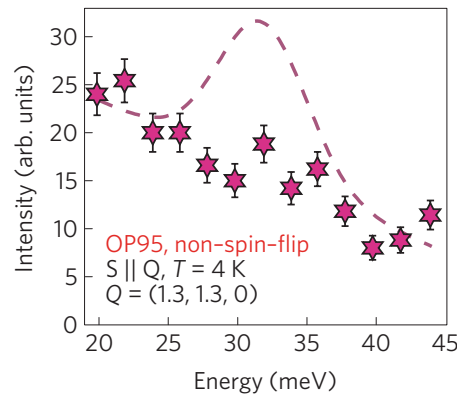

f

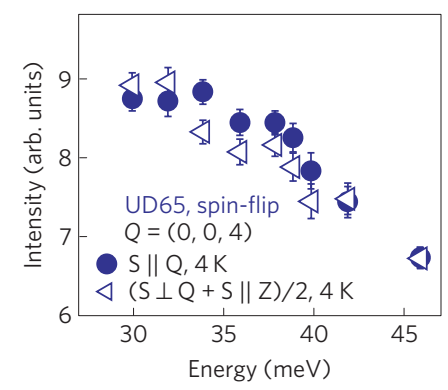

g

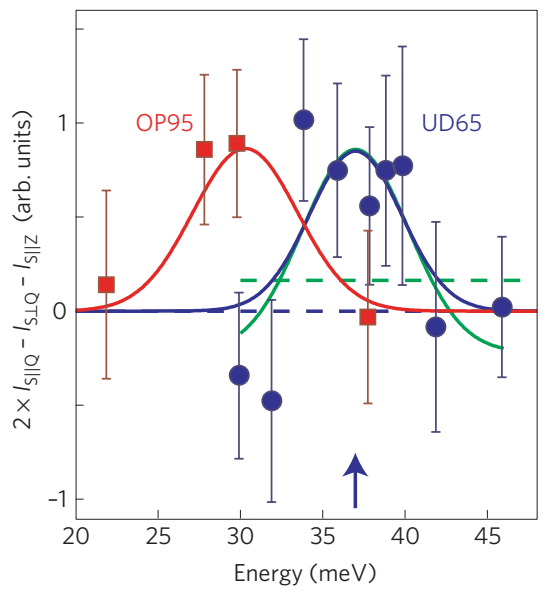

h

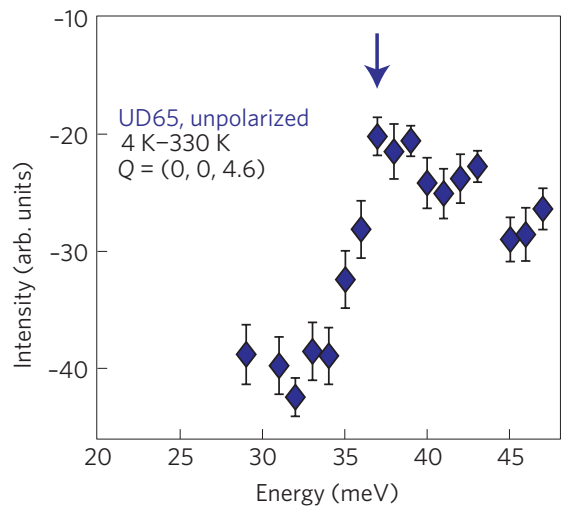

Figure 4 | Magnetic origin verified by spin-polarized measurements. a, Spin-flip spectra at $\mathbf{Q}=(0.05,0.05,4.4)$ for OP95. Filled symbols are measured with the initial neutron spin polarization (S) parallel to $\mathbf{Q}$, a geometry in which all magnetic fluctuations are probed. Open symbols are the average of intensities measured with $\mathbf{S}$ in the horizontal scattering plane but perpendicular to $\mathbf{Q}(\mathbf{S} \perp \mathbf{Q})$ and with $\mathbf{S}$ vertical $(\mathbf{S} \| \mathbf{Z})$, which measures only half of the total magnetic signal (Supplementary Fig. S5, shows that $I_{S \perp Q}$ and $I_{S \| Z}$ are the same within the error, consistent with the system's tetragonal symmetry). $\mathbf{b}, \mathbf{c}$, Intensity difference between $4 \mathrm{~K}$ and $250 \mathrm{~K}$ for OP95 measured in the $\mathbf{S} \| \mathbf{Q}$ spin-flip geometry at $\mathbf{Q}=(0.05,0.05,4.4)$ and $(1.3,1.3,0)$, respectively. Solid lines are Gaussian fits assuming a common width and baseline. d,e, Non-spin-flip intensity at $4 \mathrm{~K}$ for sample OP95 at $\mathbf{Q}=(0.05,0.05,4.4)$ and $(1.3,1.3,0)$. Dotted lines illustrate the size of the non-spin-flip nuclear (phonon) signal that would be required to produce the peaks in $\mathbf{b}, \mathbf{c}$ via polarization leakage given the instrumental flipping ratio of $\sim 10$. $\mathbf{f}$, Spin-flip data at $\mathbf{Q}=(0,0,4)$ for UD65. $\mathbf{g}$, Magnetic signal extracted from polarization analysis of the $4 \mathrm{~K}$ data in $\mathbf{a}$ and $\mathbf{f}$. Solid blue line is the best Gaussian fit to the data for UD65 assuming zero offset. Solid and dashed green lines are best Gaussian and constant fits which allow for a non-zero offset. Red line is adapted from the fit in $\mathbf{b}$ without the linear baseline. A statistical analysis of the data is presented in the Supplementary Information. h, Unpolarized neutron data for UD65 adapted from Fig. 1b to directly demonstrate that the magnetic signal in $\mathbf{g}$ occurs at the peak position of the unpolarized result for closely similar values of $\mathbf{Q}$. Error bars represent statistical uncertainty ( $1 \mathrm{~s}$.d.).

Our results are consistent with the orbital-current theory. We note, however, that a distinctly different possibility consistent with the very weak dispersion is that the excitations are related to intrinsic inhomogeneity in the local electronic environment ${ }^{35,36}$. It has been proposed that such inhomogeneity can give rise to local 'edge modes' that are partially magnetic ${ }^{35}$.

Our data reveal an intriguing connection between the pseudogap excitations and the conventional antiferromagnetic fluctuations at $\mathbf{q}_{\mathrm{AF}}$. Initial evidence comes from the prior observation for OP95 (ref. 24) that the resonance occurs at an energy which is indistinguishable from that of the high-energy pseudogap excitation, which is confirmed with improved precision in Supplementary Figs S1a and S2. A local intensity maximum at $\mathbf{q}_{\mathrm{AF}}$ is also found for the low-energy excitation in OP95 (Supplementary Fig S1b), but the relatively weak signal does not allow a detailed study. Even though there exists no clear resonance (distinct intensity change) across $T_{c}$ in UD65, we observe an enhanced response at $\mathbf{q}_{\mathrm{AF}}$ at $39 \mathrm{meV}$, the energy of the pseudogap excitation (Fig. 5a). Figure $5 \mathrm{~b}$ provides a detailed view of the response near $\mathbf{q}_{\mathrm{AF}}$ along $\mathbf{a}^{*}$. For YBCO, this momentum direction is optimal for observing the 'hourglass' dispersion of the antiferromagnetic fluctuations in the superconducting state $^{37}$. Indeed, we find initial evidence for a similar concave dispersion near $\mathbf{q}_{\mathrm{AF}}$ in $\mathrm{Hg} 1201$, with a maximum energy that is indistinguishable from that of the lower pseudogap excitation. The signal amplitudes of the antiferromagnetic fluctuations, determined from momentum scans (which are insensitive to the pseudogap excitations because of the weak dispersion), are comparable to those of the pseudogap excitations in $\mathrm{Hg} 1201$, and to those of antiferromagnetic fluctuations in other cuprates (for example, YBCO). Moreover, the signal that peaks at $\mathbf{q}_{\mathrm{AF}}$ exhibits maxima at approximately the same energies as the pseudogap excitations 
a

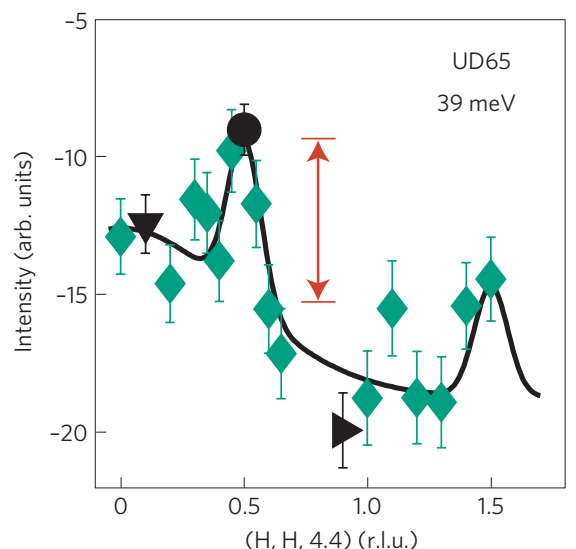

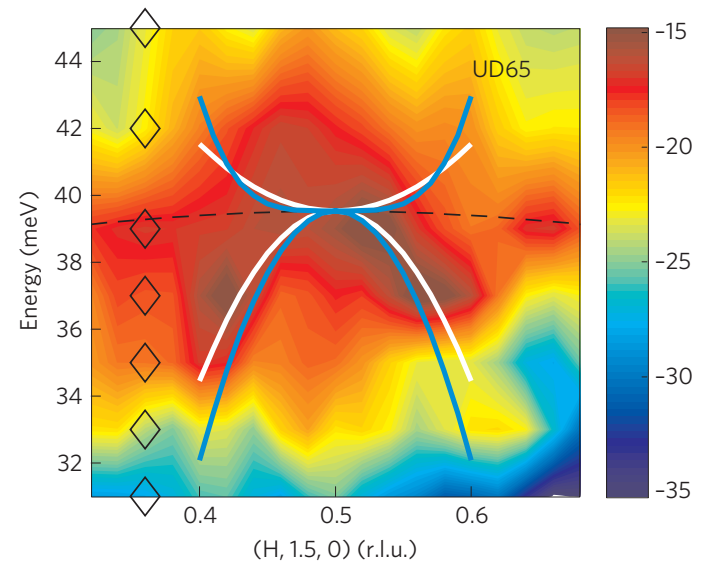

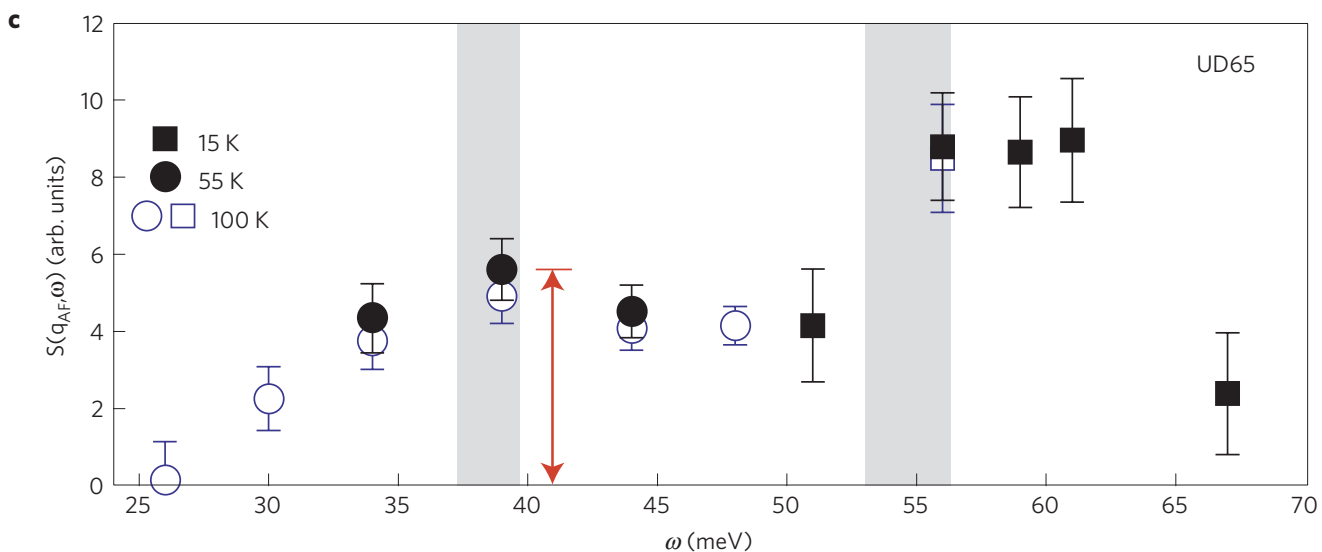

Figure 5 | Mixing between pseudogap excitations and antiferromagnetic spin fluctuations. a, Intensity difference between $4 \mathrm{~K}$ and $300 \mathrm{~K}$ at $\omega=39$ meV for UD65. Solid line is a guide to the eye, and comprises a decrease of the signal that emanates from $q=0$ towards large $Q$ and additional intensities at $\mathbf{q}_{\mathrm{AF}}$. Black symbols are data shared in common with the energy scans in Supplementary Fig. S6a. Error bars represent statistical uncertainty (1 s.d.). b, Colour representation of the intensity difference between $4 \mathrm{~K}$ and $330 \mathrm{~K}$ for UD65. Data are smoothed along the horizontal axis to enhance visual inspection (Supplementary Fig. S7). Diamond symbols indicate the energy positions of the underlying momentum scans. Solid lines illustrate the typical 'hourglass' dispersion of the spin fluctuations near $\mathbf{q}_{\mathrm{AF}}$ in $\mathrm{YBCO}$, adapted from ref. 37 (blue) and ref. 46 (white). Dashed line indicates the dispersion of the pseudogap excitation. c, Amplitude of antiferromagnetic response (peak at $(0.5,0.5)$, as indicated by the arrows) for UD65 as a function of energy ( $\omega$ ), measured on the spectrometers $2 \mathrm{~T}$ (circles) and PUMA (squares). Error bars represent fit uncertainty for signal amplitudes observed in individual scans (Supplementary Fig. $\mathbf{S 6} 6, \mathrm{c}$ ). Shaded areas illustrate the full dispersion of the pseudogap excitations (maximal dispersion energies reached at $\mathbf{q}_{\mathrm{AF}}$ ).

(Supplementary Fig. S6, summarized in Fig. 5c). Evidently, the two types of excitations mix, even though they seem to have rather different physical origins: whereas the fluctuations near $\mathbf{q}_{\mathrm{AF}}$ are generally thought to arise from copper spin moments, the weakly-dispersive pseudogap excitations seem to require the explicit consideration of oxygen orbitals and are best explained by the orbital-current theory.

Understanding the confluence of the two types of magnetic excitation will require a unifying theoretical approach. In the orbital-current theory, the superconducting pairing is the result of quantum critical fluctuations associated with the discrete pseudogap order parameter ${ }^{38}$, and antiferromagnetic correlations have not yet been included. On the other hand, theories in which the pairing is mediated by antiferromagnetic fluctuations ${ }^{39-41}$ have generally ignored the possibility that the pseudogap regime is a genuine new phase. As the superconductivity is an instability of the peculiar 'normal' state, our results imply that even if antiferromagnetic fluctuations play a role in bringing about superconductivity in the cuprates, they must not be thought of as mere remnants of the Mott-insulating state, but rather as fundamentally modified by the pseudogap state that is characterized by weakly-dispersive excitations. In fact, the size of the superconducting gap $(\Delta)$ seems to be defined already at $T^{*}$ : the magnetic resonance energy in unconventional superconductors has been shown to be universally proportional to $\Delta$ (ref. 21 ) and, in the model compound Hg1201, the resonance occurs at the same energy as the high-energy pseudogap excitation.

Bearing in mind that the pseudogap excitations and the antiferromagnetic fluctuations in Hg1201 occur at the same energy, we note that there might exist a correspondence between the magnetic energy scales of single-layer Hg1201 and double-layer YBCO, two cuprates with similar values of $T_{c, \max }$ and $\Delta$, and with well-defined resonances at $\mathbf{q}_{\mathrm{AF}}$ near optimal doping ${ }^{13,15-17,30}$. In YBCO, the presence of two resonances in the $30-60 \mathrm{meV}$ range has been interpreted as due to the interaction between the two adjacent $\mathrm{CuO}_{2}$ layers in the same primitive cell ${ }^{42}$. Surprisingly, we find that the energies of the pseudogap excitations in UD65 Hg1201 $\left(39 \pm 2 \mathrm{meV}\right.$ and $56 \pm 2 \mathrm{meV}$ at $\mathbf{q}_{\mathrm{AF}}$ ) are equal within the error to those of the odd $(\approx 37 \mathrm{meV})$ and even $(\approx 55 \mathrm{meV})$ parity resonances in YBCO with a similar $T_{\mathrm{c}}(\approx 63 \mathrm{~K}$; ref. 43$)$. This observation also holds for the high-energy mode of OP95 Hg1201 (55 $\pm 2 \mathrm{meV}$ at $\left.\mathbf{q}_{\mathrm{AF}}\right)$, but not for the corresponding low-energy mode $(32 \pm 3 \mathrm{meV}$ at $\left.\mathbf{q}_{\mathrm{AF}}\right)$ : in nearly optimally-doped $\mathrm{YBCO}\left(T_{\mathrm{c}} \approx 89 \mathrm{~K}\right)$, the two resonance energies are about 53 and $41 \mathrm{meV}$ (ref. 42). 
The pseudogap excitations should be most easily discernable in compounds in which the $q=0$ order is prominent, and so far they have been reported only for $\mathrm{Hg} 1201$. The well-studied single-layer materials $(\mathrm{La}, \mathrm{Nd}, \mathrm{Sr}, \mathrm{Ba})_{2} \mathrm{CuO}_{4}$ possess a relatively low $T_{c, \text { max }}$ of about $40 \mathrm{~K}$ and have long been known to exhibit an instability towards broken translational symmetry (spin/charge 'stripe' order) well below $T^{*}$ (ref. 18). The lack of evidence of pseudogap excitations in these compounds probably results from a competition between the two types of order ${ }^{34}$.

On the other hand, it should be possible to observe the pseudogap excitations in YBCO $\left(T_{\mathrm{c}, \max } \approx 93 \mathrm{~K}\right)$. At low doping, near the onset of superconductivity, neutron diffraction measurements have revealed a quasi-elastic signal consistent with a transition to long-range spin-density-wave order as $T \rightarrow 0$ (ref. 20). The spindensity-wave and $q=0$ orders are associated with very different wave vectors and seem to compete in the deeply underdoped regime ( $p<0.09$; ref. 44$)$, whereas the $q=0$ order is found to dominate at higher doping ${ }^{6}$, where the pseudogap excitations are most likely to be found. Material-specific differences, such as the more complicated double-layer structure of YBCO, can be expected to cause variations in the number of pseudogap excitations and in their strength relative to antiferromagnetic fluctuations. Analogous to the situation for single-layer LSCO and Hg1201, the pseudogap magnetism in the double-layer compounds might eventually be most clearly revealed in $\mathrm{HgBa}_{2} \mathrm{CaCu}_{2} \mathrm{O}_{8+\delta}\left(T_{c, \max } \approx 124 \mathrm{~K}\right.$ (ref. 45), the highest value for all double-layer compounds) once sizeable single crystals become available.

\section{Received 14 November 2011; accepted 16 February 2012; published online 18 March 2012}

\section{References}

1. Varma, C. M. Non-Fermi-liquid states and pairing instability of a general model of copper oxide metals. Phys. Rev. B 55, 14554-14580 (1997).

2. Chakravarty, S., Laughlin, R. B., Morr, D. K. \& Nayak, C. Hidden order in the cuprates. Phys. Rev. B 63, 094503 (2001).

3. Kivelson, S. A. et al. How to detect fluctuating stripes in the high-temperature superconductors. Rev. Mod. Phys. 75, 1201-1241 (2003).

4. Zaanen, J. in 100 Years of Superconductivity (eds Rogalla, H. \& Kes, P. H.) (Taylor \& Francis, 2012).

5. Kaminski, A. et al. Spontaneous breaking of time-reversal symmetry in the pseudogap state of a high- $T_{\mathrm{c}}$ superconductor. Nature 416, 610-613 (2002).

6. Fauqué, B. et al. Magnetic order in the pseudogap phase of high- $T_{c}$ superconductors. Phys. Rev. Lett. 96, 197001 (2006).

7. Li, Y. et al. Unusual magnetic order in the pseudogap region of the superconductor $\mathrm{HgBa}_{2} \mathrm{CuO}_{4+\delta}$. Nature 455, 372-375 (2008).

8. Li, Y. et al. Magnetic order in the pseudogap phase of $\mathrm{HgBa}_{2} \mathrm{CuO}_{4+\delta}$ studied by spin-polarized neutron diffraction. Phys. Rev. B 84, 224508 (2011).

9. Xia, J. et al. Polar Kerr-effect measurements of the high-temperature $\mathrm{YBa}_{2} \mathrm{Cu}_{3} \mathrm{O}_{6+x}$ superconductor: Evidence for broken symmetry near the pseudogap temperature. Phys. Rev. Lett. 100, 127002 (2008).

10. He, R-H. et al. From a single-band metal to a high-temperature superconductor via two thermal phase transitions. Science 331, 1579-1583 (2011).

11. Lawler, M. J. et al. Intra-unit-cell electronic nematicity of the high- $T_{\mathrm{c}}$ copper-oxide pseudogap states. Nature 466, 347-351 (2010).

12. Birgeneau, R. J.et al. Antiferromagnetic spin correlations in insulating, metallic, and superconducting $\mathrm{La}_{2-x} \mathrm{Sr}_{x} \mathrm{CuO}_{4}$. Phys. Rev. B 38, 6614-6623 (1988).

13. Rossat-Mignot, J. et al. Neutron scattering study of the $\mathrm{YBa}_{2} \mathrm{Cu}_{3} \mathrm{O}_{6+x}$ system. Physica C 185-189, 86-92 (1991).

14. Cheong, S-W. et al. Incommensurate magnetic fluctuations in $\mathrm{La}_{2-x} \mathrm{Sr}_{x} \mathrm{CuO}_{4}$. Phys. Rev. Lett. 67, 1791-1794 (1991).

15. Mook, H. A., Yethiraj, M., Aeppli, G., Mason, T. E. \& Armstrong, T. Polarized neutron determination of the magnetic excitations in $\mathrm{YBa}_{2} \mathrm{Cu}_{3} \mathrm{O}_{7}$ Phys. Rev. Lett. 70, 3490-3493 (1993).

16. Bourges, P., Regnault, L. P., Sidis, Y. \& Vettier, C. Inelastic-neutron-scattering study of antiferromagnetic fluctuations in $\mathrm{YBa}_{2} \mathrm{Cu}_{3} \mathrm{O}_{6.97}$. Phys. Rev. B 53, 876-885 (1996).

17. Fong, H. F., Keimer, B., Reznik, D., Milius, D. L. \& Aksay, I. A. Polarized and unpolarized neutron-scattering study of the dynamical spin susceptibility of $\mathrm{YBa}_{2} \mathrm{Cu}_{3} \mathrm{O}_{7}$. Phys. Rev. B 54, 6708-6720 (1996).

18. Tranquada, J. M., Sternlieb, B. J., Axe, J. D., Nakamura, Y. \& Uchida, S. Evidence for stripe correlations of spins and holes in copper oxide superconductors. Nature 375, 561-563 (1995).

19. Birgeneau, R. J., Stock, C., Tranquada, J. M. \& Yamada, K. Magnetic neutron scattering in hole-doped cuprate superconductors. J. Phys. Soc. Jpn 75, 111003 (2006).
20. Haug, D. et al. Neutron scattering study of the magnetic phase diagram of underdoped $\mathrm{YBa}_{2} \mathrm{Cu}_{3} \mathrm{O}_{6+x}$. New J. Phys. 12, 105006 (2010).

21. Yu, G., Li, Y., Motoyama, E. M. \& Greven, M. A universal relationship between magnetic resonance and superconducting gap in unconventional superconductors. Nature Phys. 5, 873-875 (2009).

22. Demler, E. \& Zhang, S-C. Quantitative test of a microscopic mechanism of high-temperature superconductivity. Nature 396, 733-735 (1998).

23. Dahm, T. et al. Strength of the spin-fluctuation-mediated pairing interaction in a high-temperature superconductor. Nature Phys. 5, 217-221 (2009).

24. Li, Y. et al. Hidden magnetic excitation in the pseudogap phase of a high- $T_{\mathrm{c}}$ superconductor. Nature 468, 283-285 (2010).

25. Varma, C. M. Theory of the pseudogap state of the cuprates. Phys. Rev. B 73, 155113 (2006).

26. Varma, C. M. Mind the pseudogap. Nature 468, 184-185 (2010).

27. Eisaki, H. et al. Effect of chemical inhomogeneity in bismuth-based copper oxide superconductors. Phys. Rev. B 69, 064512 (2004).

28. Barišic̀, N. et al. Demonstrating the model nature of the high-temperature superconductor $\mathrm{HgBa}_{2} \mathrm{CuO}_{4+\delta}$. Phys. Rev. B 78, 054518 (2008).

29. Zhao, X. et al. Crystal growth and characterization of the model high-temperature superconductor $\mathrm{HgBa}_{2} \mathrm{CuO}_{4+\delta}$. Adv. Mater 18, 3243-3247 (2006).

30. $\mathrm{Yu}, \mathrm{G}$. et al. Magnetic resonance in the model high-temperature superconductor $\mathrm{HgBa}_{2} \mathrm{CuO}_{4+\delta}$. Phys. Rev. B 81, 064518 (2010)

31. Li, Y., Egetenmeyer, N., Gavilano, J. L., Barišic̀, N. \& Greven, M. Magnetic vortex lattice in $\mathrm{HgBa}_{2} \mathrm{CuO}_{4+\delta}$ observed by small-angle neutron scattering. Phys. Rev. B 83, 054507 (2011).

32. Ikeda, H. \& Hutchings, M. T. Spin wave excitations in a two-dimensional Ising-like antiferromagnet, $\mathrm{Rb}_{2} \mathrm{CoF}_{4}$. J. Phys. C 11, L529 (1978).

33. He, Y. \& Varma, C. M. Collective modes in the loop ordered phase of cuprate superconductors. Phys. Rev. Lett. 106, 147001 (2011).

34. Balédent, V. et al. Two-dimensional orbital-like magnetic order in the high-temperature $\mathrm{La}_{2-x} \mathrm{Sr}_{x} \mathrm{CuO}_{4}$ superconductor. Phys. Rev. Lett. 105, 027004 (2010).

35. Martin, I., Kaneshita, E., Bishop, A. R., McQueeney, R. J. \& Yu, Z. G. Vibrational edge modes in intrinsically heterogeneous doped transition metal oxides. Phys. Rev. B 70, 224514 (2004).

36. Tahir-Kheli, J. \& Goddard, W. A. III Origin of the pseudogap in high-temperature cuprate superconductors. J. Phys. Chem. Lett. 2, 2326-2330 (2011).

37. Hinkov, V. et al. Spin dynamics in the pseudogap state of a high-temperature superconductor. Nature Phys. 3, 780-785 (2007).

38. Aji, V., Shekhter, A. \& Varma, C. M. Theory of the coupling of quantum-critical fluctuations to fermions and $d$-wave superconductivity in cuprates. Phys. Rev. B 81, 064515 (2010).

39. Abanov, Ar., Chubukov, A. V. \& Schmalian, J. Quantum-critical theory of the spin-fermion model and its application to cuprates: Normal state analysis. Adv. Phys. 52, 119-218 (2003).

40. Moriya, T. \& Ueda, K. Antiferromagnetic spin fluctuation and superconductivity. Rep. Prog. Phys. 66, 1299-1341 (2003).

41. Barzykin, V. \& Pines, D. Universal behaviour and the two-component character of magnetically underdoped cuprate superconductors. Adv. Phys. 58, 1-65 (2009).

42. Pailhès, S. et al. Resonant magnetic excitations at high energy in superconducting $\mathrm{YBa}_{2} \mathrm{Cu}_{3} \mathrm{O}_{6.85}$. Phys. Rev. Lett. 93, 167001 (2004).

43. Pailhès, S. et al. Doping dependence of bilayer resonant spin excitations in (Y, Ca) $\mathrm{Ba}_{2} \mathrm{Cu}_{3} \mathrm{O}_{6+x}$. Phys. Rev. Lett. 96, 257001 (2006).

44. Balédent, V.et al. Evidence for competing magnetic instabilities in underdoped $\mathrm{YBa}_{2} \mathrm{Cu}_{3} \mathrm{O}_{6+x}$. Phys. Rev. B 83, 104504 (2011).

45. Tsuei, C. C., Gupta, A., Trafas, G. \& Mitzi, D. Superconducting mercury-based cuprate films with a zero-resistance transition temperature of $124 \mathrm{~K}$. Science 263, 1259-1261 (1994)

46. Reznik, D. et al. Dispersion of magnetic excitations in optimally doped superconducting $\mathrm{YBa}_{2} \mathrm{Cu}_{3} \mathrm{O}_{6.95}$. Phys. Rev. Lett. 93, 207003 (2004).

\section{Acknowledgements}

We thank C. M. Varma, A. R. Bishop and B. Keimer for stimulating discussions. This research was supported by the US Department of Energy, office of Basic Energy Sciences. X.Z. acknowledges support by the National Natural Science Foundation, China. Y. Li acknowledges support from the Alexander von Humboldt Foundation.

\section{Author contributions}

M.G., P.B. and Y.L. planned the project. Y.L., G.Y., M.K.C., V.B. and Yangmu L. performed the neutron scattering experiments. Y.L., N.B. and X.Z. characterized and prepared the samples. P.S., R.A.M., K.H., Y.S. and P.B. were local contacts for the neutron scattering experiments. Y.L. and M.G. analysed the data and wrote the manuscript with input from all coauthors.

\section{Additional information}

The authors declare no competing financial interests. Supplementary information accompanies this paper on www.nature.com/naturephysics. Reprints and permissions information is available online at www.nature.com/reprints. Correspondence and requests for materials should be addressed to M.G. 